\title{
On the Performances of the Routing Protocols in MANET: Classical Versus Self-organized Approaches
}

\author{
Fabrice Theoleyre and Fabrice Valois \\ CITI, INRIA ARES, INSA Lyon, \\ 21 av Jean Capelle, 69621 Villeurbanne Cedex, France \\ \{fabrice.theoleyre, fabrice.valois\}@insa-lyon.fr
}

\begin{abstract}
Mobile Ad Hoc Networks (MANET) are spontaneous wireless networks of mobile nodes without any fixed infrastructure. MANET are promised to a large spectrum of military or civilian utilizations. Routing is a key topic in such networks: overhead must be minimized, optimizing the delay and reducing the packet losses. Several routing protocols were proposed in the literature but, recently, new routing protocols based on a self-organization like Virtual Structure Routing (VSR) were proposed. VSR is based on a self-organized structure with an important stability and persistence. In this paper, we aim to quantify the contribution of the self-organization on the routing behavior and performances. We oppose VSR as a self-organized protocol to the classical one: reactive (AODV), proactive (OLSR) and clustered (CBRP). The impact of the mobility and the density, the horizontal and the vertical scalabilities are studied.
\end{abstract}

\section{Introduction}

Mobile Ad Hoc Networks (MANET) are literally networks ready to work. All terminals can communicate with other nodes via wireless communications: MANET are spontaneous networks, without any fixed infrastructure. The network must function autonomously, without any human intervention: the self-organization property is vital. In consequence, the nodes must collaborate to set up all network functions fulfilled traditionally by specialized devices. Moreover, a source can be not in the range of the destination: nodes must relay the packets from a source to a destination: the network is multihops. Thus, a distributed routing algorithm must be proposed. Efficient routes must be computed distributively, each node being both router and client. Moreover, all nodes are mobile, creating topology changes. Hence, the network must continuously adapt its knowledge of the topology in order to maintain efficient routes. Ad hoc networks can be connected to the Internet via an Access Point, creating multihops cellular networks.

MANET constitute a wide domain to study. All classical solutions must be re-conceived because of the particular constraints of MANET. The radio links offer a low bandwidth, and create an important instability: fading and multi 
paths create brutal changes in the radio topology. In the same way, the packet losses are frequent because of collisions. Moreover, MANET are constituted by a collection of different embedded terminal: constraints in energy, CPU and memory are important. Besides, the network is heterogeneous: laptops cohabit with PDA or wired workstations. Thus, propositions must take into account such an heterogeneity, reflecting the different behavior and capacities of the nodes. The load must be balanced efficiently among the nodes, according to their capabilities.

According to us, the self-organization gives multiple answers to the key problems described above. The self-organization is for us the simplification of the topology to facilitate the exploitation of an ad hoc network. The self-organization structures the network, creates a hierarchy, and reduces the topology changes in creating a virtual topology. The self-organization must take into account the network heterogeneity: a node with more power-energy or with a low mobility will contribute more in the network management. In [9], we proposed a virtual topology of self-organization reflecting good properties of stability and persistence. Thus, a routing protocol (Virtual Structure Routing, VSR [1]) benefiting from it was proposed. In this article, we propose to compare a self-organized protocol like VSR with the classical routing protocols existing for MANET. More specifically, one reactive protocol (AODV), one proactive protocol (OLSR) and one hierarchical protocol (CBRP) will be evaluated and simulated. Simulations will measure the performances according to several criteria and environment parameters.

In the first section, we will present a short related work about the selforganization, useful to understand finely the benefits of a self-organization for routing. In the section 3, an overview of the different classes of routing protocols will be given. The section 4 will present the performances evaluation of the different routing classes in MANET. Finally, the section 5 will conclude the article and give some perspectives.

\section{Virtual Structures of Self-organization}

\section{$2.1 \quad$ Related Work}

Backbone. A backbone helps to optimize the traffic exchange, and to reduce the impact of the flooding, minimizing the broadcast storm problem. Only backbone members relay a control packet, reducing the load on the medium.

MANET could be modeled with the graph theory: each terminal is represented by a vertex, and there exists one edge between two vertices if the corresponding terminals have a radio link with each other. In the graph theory, a backbone could be modeled as a Minimum Connected Dominating Set (MCDS): each node is either in the MCDS, or neighbor of at least one vertex of the MCDS. Moreover, the MCDS forms a connected structure of minimal cardinality. MCDS being NPcomplete 4], some heuristics must be proposed. A Connected Dominating Set (CDS) is a MCDS without the constraint of minimal cardinality. We extend this notion to a k-CDS: the maximal distance from one node to the CDS is inferior to $k$ hops. 
Several articles propose to construct distributively a CDS, minimizing the cardinality [1,3]. Generally, the algorithms are divided in two major steps: the creation of the dominating set (each node is neighbor of one node of the dominating set), and then its interconnection. A node can be dominator (member of the CDS), dominatee (the node has a neighbor in the CDS) or idle. During the first step, an idle node with the highest weight among its idle neighbors becomes dominator. The degree or the identifier can represent the weight. An idle node neighbor of a dominator becomes dominatee. The second step interconnects the dominators. However, a minimal number of dominators must be chosen. [3] proposes an iterative exploration, starting from the leader, choosing during each iteration to color the locally best dominatee. However, such an exploration requires an high delay. [1] proposes a best-effort approach: a dominator connects itself to any dominator already connected and at most 2 hops far. The delay is reduced. No maintenance procedure is described although topology changes require to update continuously the structure.

12 is, to the best of our knowledge, the only localized algorithm constructing a CDS. A node is colored dominatee if the following rule is valid: no couple of my neighbors are not directly connected. Else, the node is dominator. This rule forms globally a CDS. The rule was extended further in: there exists a connected set of neighbors of higher weight which are a dominating set of my whole neighborhood. This CDS was proposed to optimize the flooding, but not the persistence of the structure. Thus, simulations show that a node changes frequently its role, creating potentially an unstable hierarchy.

Clusters. Clusters allow to divide the network, creating a hierarchy. Clusters constitute natural services areas. The constraint of a cluster can be its diameter: the maximal distance from one node to another node of the same cluster is at most 3 hops. A leader called clusterhead can also be elected and maintained in the cluster. In this case, the radius represents the cluster constraint.

[7. presents an algorithm widely used in the literature to form clusters. Each node initiates a neighborhood discovering. A node with the highest weight among its neighbors without clusterhead is elected clusterhead. Its neighbors join its cluster. During the maintenance, the clusterhead is no longer maintained. When a node detects that the diameter constraint is violated, the nodes decide distributively how to split the cluster. The knowledge of the 2-neighborhood is required. If a clusterhead is maintained, the maintenance can use the radius constraint.

2. proposes the creation of k-clusters: each node is at most $k$ hops far from its clusterhead. The algorithm could be divided in two waves: the first wave propagates the highest ids, and the second wave propagates the lowest ids. However, no maintenance procedure is described.

\subsection{The Virtual Structure Used by VSR}

9] presents a virtual structure combining both a backbone and clusters. The structure is fully integrated in order to reduce the overhead: the algorithms to maintain the backbone and the clusters share some information. This virtual structure is used by VSR [11] to provide a new self-organized routing protocol. 

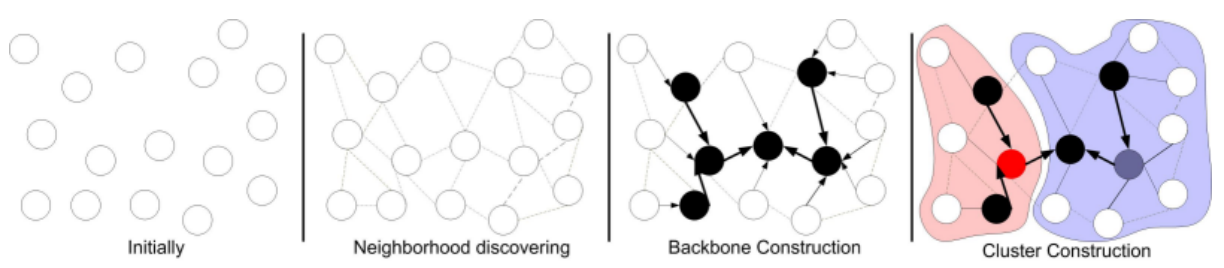

Fig. 1. Virtual structure construction

First, a $\mathrm{k}_{c d s}$-neighborhood discovering is triggered: all the nodes exchange periodically hello packets to discover all the nodes at most $\mathrm{k}_{c d s}$ hops far, their identity, weight, state... Then, the algorithm constructs a $\mathrm{k}_{c d s}$-CDS in electing dominators and interconnecting them, forming a backbone: each node is at most $k$ hops far from the backbone, $\mathrm{k}_{c d s}$ being a parameter of the protocol. The backbone construction is triggered by one or several leaders. In an hybrid network, the gateway to the Internet should act as leader. Else, a leader must elected distributively. Finally when the $\mathrm{k}_{c d s}$-CDS is locally constructed and based on the sub-graph of dominators, clusterheads are elected such than $\mathrm{k}_{\text {cluster-clusters }}$ are built.

However, the radio topology changes continuously in MANET. Thus, 9] details event-driven procedures to maintain an efficient virtual structure. All the nodes in the network maintain a parent in the backbone, so that each node is at most $k$ hops far from the backbone. Besides, procedures allow to verify distributively the backbone connectivity, and eventually to reconnect it. In the same way, distributed procedures allow to maintain the dominance property of the clusters.

This virtual structure of self-organization was proved to present interesting properties of self-stabilization [10. Starting from any initial state, potentially corrupted, the algorithms converge to a valid state in a finite time. Moreover, local changes in the radio topology impacts only locally the virtual structure, improving both the robustness and the stability.

\section{Routing Protocols}

\subsection{Flat Routing}

Flat routing protocols do not introduce a hierarchy among the nodes: all nodes are equal and participate to the routing process. Classically, they can be divided in the reactive and the proactive classes.

Reactive. Reactive protocols propose to discover routes on demand. In AODV (Ad Hoc On demand Distance Vector Routing) [8], when a node wants to send a Data Packet, and no route is present, it sends a Route Request in broadcast. When a node receives a Route Request, it adds an entry in its routing table pointing to the source of the request via the previous hop. Then, if no route to 
the searched node is known, it forwards the Route Request. Else, it generates a Route Reply, sent along the inverse route. The Route Reply is forwarded in unicast and creates an entry in the routing table of each intermediary node pointing to the destination. A Route Error is created if a route is broken. This packet sent to the source allows to delete failed entries in the routing tables. The route discovering introduces a latency, but the overhead is minimized. Flooding used for the route discovering can create a broadcast storm.

Proactive. Proactive protocols propose to maintain all along the time all the routes. If a node floods periodically topology packets in the network, an heavy load on the radio medium is created and collisions occur. OLSR (Optimized Link State Routing) 5] proposes to limit the impact of the flooding. Each node selects a Multi Point Relays (MPR) set: the MPRs are neighbors and cover all the 2-neighborhood. When a node sends a Topology Packet, only its MPRs forward it. Recursively, only the MPR of the MPR will relay the control packets, reducing the overhead. However, the overhead remains important and could be useless if a node communicates only with a few destinations, using only a few routes.

\subsection{Hierarchical Routing}

To overcome the problems of flat routing, clustered routing protocols were proposed. CBRP (Cluster Based Routing Protocol) [6] is based on a hierarchy of clusters and clusterheads. Each node sends periodically Hellos containing the list of neighbors and the list of adjacent clusters (the clusterheads of neighbors). The protocol is reactive: a node sends a route discovering among the clusterheads and the gateways. The reply is sent along the inverse route, each clusterhead trying to bypass itself in the route if possible. The route is registered in the packet, CBRP being a source routing protocol. However, the topology is only used for the route discovering. CBRP is finally a flat routing protocol, the route being constituted only by a list of individual nodes.

\subsection{Routing on a Self-organization}

11] proposed a leader-based framework of routing protocol, VSR (Virtual Structure Routing), based on the virtual structure of self-organization described in the section 2.2. A proactive routing protocol is implemented in a cluster: each node knowns proactively all the routes of its cluster. For the inter-cluster routing, a route is discovered on demand and is based on the cluster id rather than on the node id in order to benefit of the virtual structure stability and robustness properties. A route is constituted by a list of clusters from the source to the destination. When a node wants to send a Data Packet and no route is known, it sends a Route Request to the nearest backbone member. This backbone member adds its cluster id in the route contained in the header of the packet and forwards the packet in multicast to other backbone members. When a backbone member receives a request and the destination is unknown, it adds its cluster id in the route if it is not present and forward the packet. In the other case, if the 
destination is present either in the neighborhood table or in the routing table, it generates a Route Reply. Then, the Route Reply is forwarded in unicast like Data Packets: a forwarder tries to reach the cluster of the route, nearest of the destination. A cluster is reachable according to the following criteria:

- A neighbor is in the searched cluster

- A neighbor is gateway for the searched cluster

- A node in the cluster is gateway for the searched cluster. This node is reachable thanks to the intra-cluster routing protocol

In consequence, the route length is optimized thanks to the local knowledge of the cluster and neighbors. Finally, a route repair mechanism is proposed: the routing algorithm is re-executed forbidding the previously failed node.

\section{Performances Comparison}

\subsection{Simulation Guidelines}

We present here results about simulations using OPNET Modeler. We used the $802.11 \mathrm{~b}$ model proposed in OPNET with a standard $300 \mathrm{~m}$ radio range, in DCF mode, without RTS/CTS. Each node moves itself according to the random waypoint mobility model, without any pause time. All results are computed with a $95 \%$ confidence interval. We consider as standard a mobility of $5 \mathrm{~m} . \mathrm{s}^{-1}$, 40 nodes, a degree (number of neighbors) of 10 , and 4 simultaneous flows. Each simulation lasts 600 seconds. The traffic generation is modeled as follows: flows of 20 data packets interspaced by $0.25 \mathrm{~s}$ are sent. For each flow, a destination and a source are randomly chosen. The inter-flow time follows an exponential distribution centered on 5 seconds to have on average the chosen number of simultaneous flows. The packet size follows an exponential distribution centered on 128 octets.

The results highlight the pertinence of using a self-organization to provide a routing scheme based on a virtual topology. We compare the performances of VSR with the performances under the same conditions of AODV, CBRP and OLSR. We simulated VSR with $k_{c d s}=1 / k_{\text {cluster }}=2$ and $k_{c d s}=2 / k_{\text {cluster }}=3$. Both configurations present similar results compared to other routing protocols, except for the overhead. Hence, we chose to represent the results only for $k_{c d s}=$ $1 / k_{\text {cluster }}=2$, except in the section dealing with the overhead.

\subsection{Performances}

Horizontal Scalability. We investigate the horizontal scalability of the different routing protocols (fig. 2). The network cardinality comprises 20 to 90 nodes. To study uniquely the impact of the number of nodes, we maintain the degree as constant. OLSR presents the lowest delay since it is a proactive protocol, and a route is found immediately. Oppositely, CBRP and AODV which are reactive present an higher delay (30ms are required when 90 nodes are present). VSR 

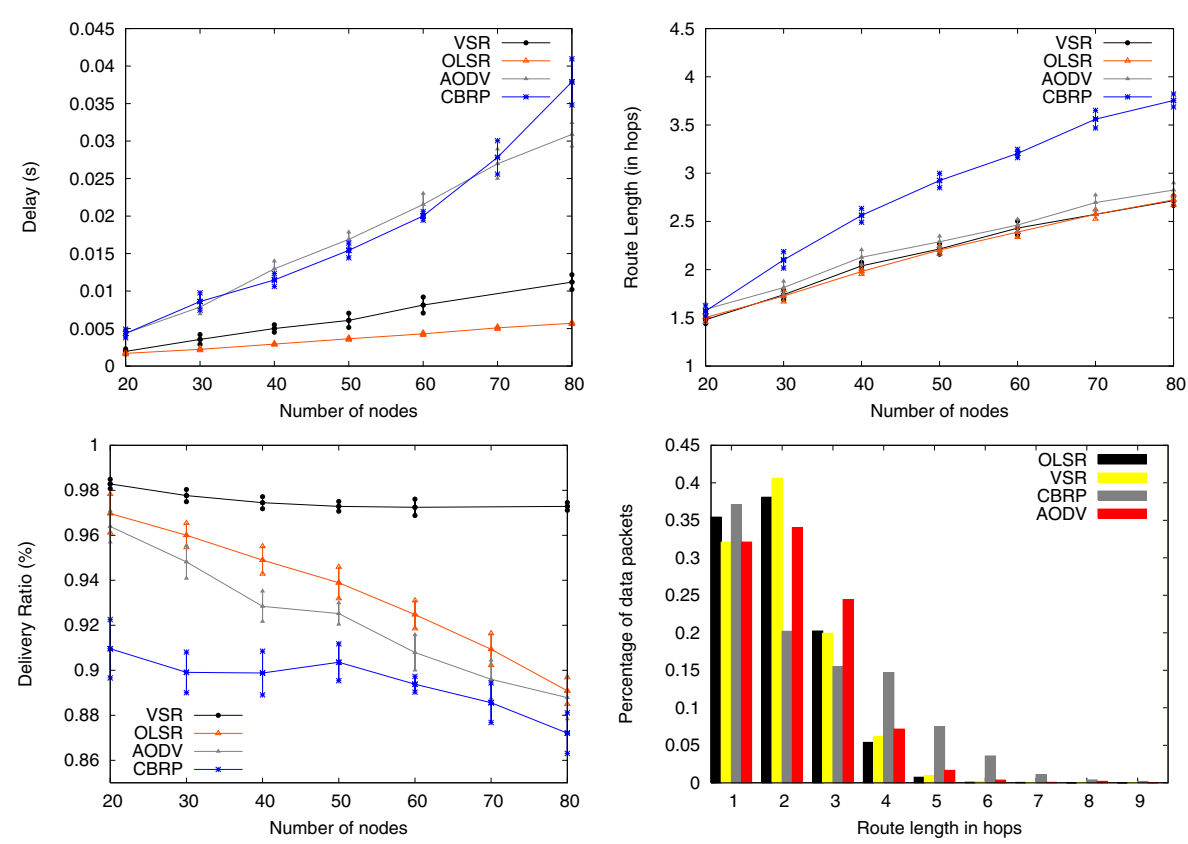

Fig. 2. Horizontal Scalabalility and the route length distribution

benefits from the self-organization: the delay is higher than OLSR, but the difference remains reasonable, even with 80 nodes. The length of the routes discovered by CBRP are longer: the Route Requests pass through the clusterheads and gateways topology. Besides, the route shortening when a clusterhead forwards a Route Reply seems to construct routes longer than the shortest routes. OLSR constructs shortest routes since each node has a complete knowledge of the topology. AODV, in spite of its reactive behavior, constructs routes near from shortest routes. VSR benefits from the virtual topology and does not create longer route although a hierarchy is used. The distribution of the route length is reported in fig 2 the proportion of the number of routes which are equals to $x$ hops is reported ( $x$ varying from 1 to 9 hops). OLSR and VSR present a very similar distribution. Since OLSR computes shortest paths, VSR achieves the discovering of short routes. AODV discovers sometimes longer routes, but the distribution is analogous to OLSR and VSR. CBRP discovers the longest routes, creating potentially more load and collisions. The delivery ratio of CBRP is the lowest (90\% with 50 nodes or more): the route is constituted by a list of nodes and is not robust. The hierarchy seems not fully exploited. AODV is a reactive protocol, and sometimes collisions occur for the Route Requests. No route is in this case discovered, and some Data Packets are dropped. In the same way, Topology Packets for OLSR can collide, creating a lack of some routes in the network. These collisions are more frequent when more nodes are present in the network: the delivery ratio decreases when the number of nodes increases. VSR combines the approaches thanks to the self-organization. Moreover, the backbone allows 

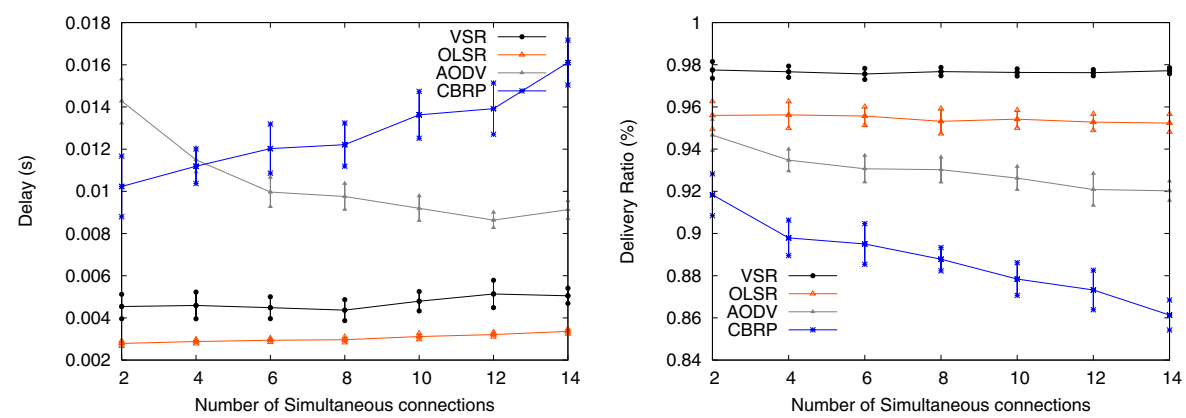

Fig. 3. Vertical Scalability

to save transmissions of control packets: collisions are less frequent. Thus, the delivery ratio is improved, and remains very stable according to the number of nodes.

Vertical Scalability. VSR and OLSR present the lowest delay, invariant according to the number of simultaneous connections (fig. (3). The delay of AODV decreases when the number of connections increases: more connections are initiated, creating more route discoverings. In consequence, a Data Packet has an higher probability to have already a route in its routing cache toward the searched destination. The delay of CBRP increases when the load in the network increases: more collisions occur because of its long routes and its unoptimized route discovering. For the route discovering, on average $20 \mathrm{~ms}$ are required for VSR, $50 \mathrm{~ms}$ for AODV and $100 \mathrm{~ms}$ for CBRP. The backbone helps greatly to optimize the route discovering. The delivery ratio of AODV, OLSR and VSR seems scalable according to the load of the network. VSR keeps on presenting the highest delivery ratio according to its stable routes thanks to the virtual topology. OLSR presents a lower delivery ratio, but stable according to the load. The delivery ratio of AODV seems to decrease slightly because of the collisions of the Route Requests.

Mobility. The impact of the mobility is represented in figure 4. The delay of OLSR remains the lowest: no delay is required to maintain or to construct a route. VSR presents a delay almost stable. The delay of AODV is higher, although almost stable. CBRP seems to suffer more from topology changes. The delivery ratio of CBRP decreases quickly when the mobility increases: at $25 \mathrm{~m} . \mathrm{s}^{-1}, 80 \%$ of the packets are received by the destination. VSR, AODV and OLSR are more scalable and present an higher delivery ratio. The packet losses increase, since rapid topology changes create route breaks. For reactive protocols, some Data packets are dropped and a new route discovering is initiated. For proactive protocols, a node must wait the next Topology Packets. VSR presents the highest delivery ratio: routes are a list of clusters, and the route of individual nodes is updated on the fly, according to the local knowledge. The routes of VSR seem more robust due to the robustness of the virtual topology. 

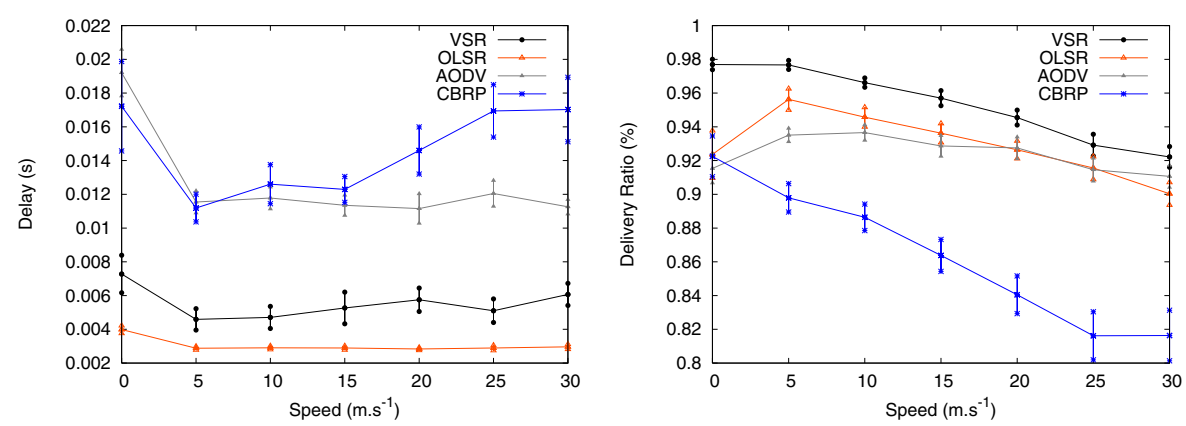

Fig. 4. Impact of the mobility
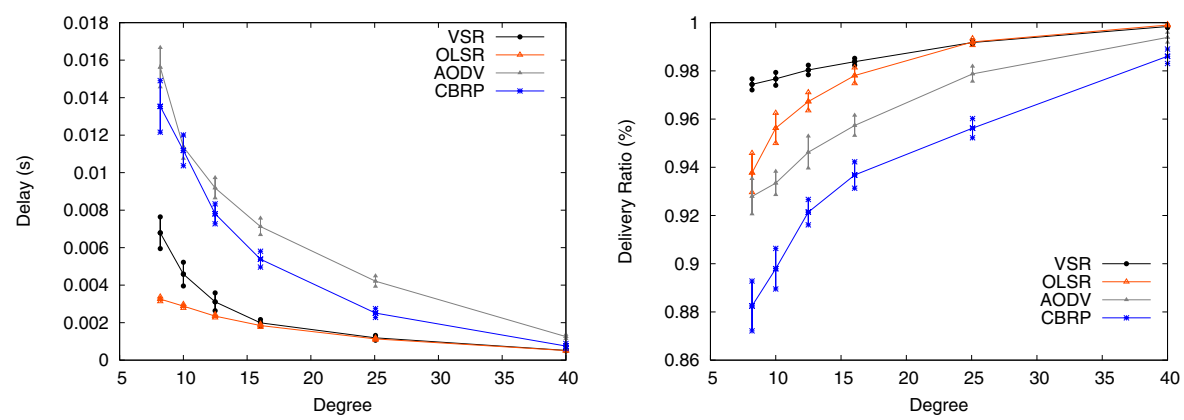

Fig. 5. Impact of the density

Density. Finally, the impact of the degree, i.e. the number of neighbors, is investigated (fig. 5). We note that the delay and the packet loss decreases when the density increases: the radius of the network is smaller, and routes are on average smaller. When the network is a single hop, all the protocols seem to react efficiently. However, for low density, reactive routing protocols seem to lose packets and suffer from an higher delay. This phenomena is perhaps because the broadcast is not reliable and many Route Requests are lost since the network is very sparse. The delay of VSR increases for very sparse networks since the Route Request must be forwarded farther. Nevertheless, the delivery ratio of VSR remains the highest among all the routing protocols: routes are stable, and the protocol seems to react efficiently to the lack of reliability of broadcasts thanks to the flooding through the virtual structure.

Overhead. Under the previous assumptions, the overhead of VSR is mainly constituted by the hellos (50\%) and the maintenance of the virtual structure $(40 \%)$. The route discovering is optimized thanks to the self-organization structure. The overhead of OLSR is mainly driven by the topology packets (78\%): a topology packet must be flooded in the whole network. The overhead of AODV is constituted by Route Request (64\%) and Route Reply (36\%). The route discovering, because of the flooding, presents an important cost. Finally, the Route Request 

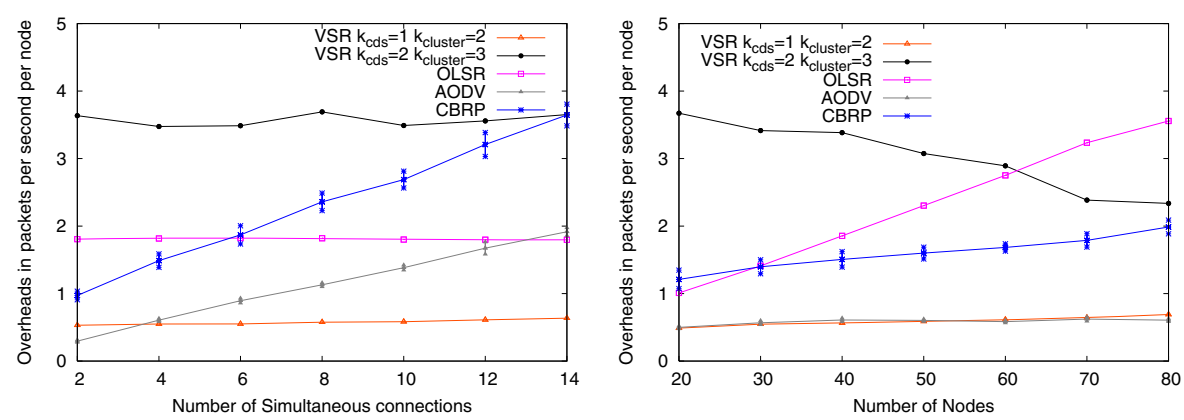

Fig. 6. Overheads of the different routing protocols

represent $90 \%$ of the overhead of CBRP. The route discovering seems not efficiently exploit the hierarchy in the network.

We also compute the total overheads of the different routing protocols according to the load of the network in packet per second per node (fig. 6). We can remark that the overhead of OLSR is stable: the proactive overhead is independent of the volume of the traffic. The overhead of AODV increases when more Data Packets must be sent. OLSR overpasses AODV when more than 14 simultaneous connections are present in the network. The overhead of CBRP increases quicker than AODV. VSR is an hybrid routing protocol and takes advantage efficiently of the virtual structure to limit the flooding. In consequence, VSR with $k_{c d s}=1 / k_{\text {cluster }}=2$ presents the lowest overhead when the load of the network exceeds a threshold. When, $k_{c d s}=2 / k_{\text {cluster }}=3$ the overhead is important because of the proactive routing protocol inside each cluster. Thus, OLSR should be implemented to reduce the control traffic. Besides, we can remark that AODV presents an efficient route discovering: if the number of connections is constant, the overhead because of the route discovering does not increase importantly. VSR is also very scalable. The route discovering process of CBRP is less efficient and presents an higher overhead. However, the overhead of OLSR increases quickly: more nodes must send Topology Packets, even if the number of connections remains constant.

Route Repairs. Finally, the impact of the route repair mechanism is measured (fig. 7). For the sake of the genericness, we do not assume the existence of a cooperation between the MAC layer and the ad hoc routing protocol layer. In the same way, the promiscuous mode is considered as non available. An Acknowledgment packet is in consequence required for each data packet. If no Acknowledgment is received after 3 transmissions, a route repair is initiated.

Route repairs introduce timeout mechanisms and retransmissions. Thus, the delay is increased for both CBRP and VSR. However, since CBRP presents already an higher delay than VSR without route repairs, CBRP keeps on presenting an higher delay. Oppositely, the delivery ratio is greatly improved. At $5 \mathrm{~m} . \mathrm{s}^{-1}$, the delivery ratio is improved by $6 \%$ for CBRP. VSR presents the highest delivery ratio, superior to $99 \%$ even with a speed of $25 \mathrm{~m} . \mathrm{s}^{-1}$. This improvement 

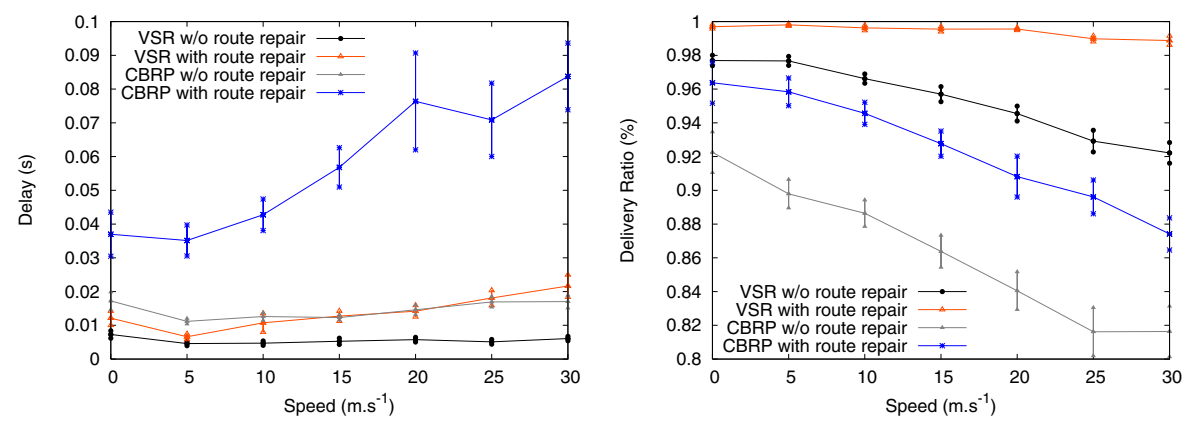

Fig. 7. Impact of the route repair mechanism of CBRP and VSR

introduces naturally an overhead: at least an Acknowledgement packet is required for each Data Packet. However, if a promiscuous mode is available, the overhead is either negligible. In a cross-layer approach with a MAC notification, it is even null.

\section{Conclusion}

In this paper, we compare the performances of a routing protocol (VSR) based on a self-organization scheme with the more classical flat approaches. VSR is based on self-organization paradigms and benefits of the stability and robustness properties of the virtual structure. It allows to offer to the routing protocol a hierarchical view of the network. VSR uses a pro-active routing inside the clusters and a reactive one for inter-cluster routing. Simulation results highlight the behavior of VSR. VSR improves the vertical and horizontal scalabilities; moreover, less packets are dropped, and the delay does not seem to suffer from the hierarchical structure. In fact, VSR appears to be a very interesting way to optimize the trade-off of proactive and reactive protocols. Based on this work, it should be interesting to use OLSR for intra-cluster routing to reduce the overhead. We are mainly interested by two perspectives. First, we have developped a testbed to validate our approach and the first results we get are very relevant. Second, because self-organization deals with better nodes and local decisions, the capacity in terms of flow should be quantified.

\section{References}

1. K. M. Alzoubi, P.-J. Wan, and O. Frieder. Distributed heuristics for connected dominating set in wireless ad hoc networks. IEEE/KICS Journal of Communications and Networks, 4(1):22-29, march 2002.

2. A. Amis, R. Prakash, T. Vuong, and D. Huynh. Max-min d-cluster formation in wireless ad hoc networks. In INFOCOM, Tel-Aviv, Israel, March 1999. IEEE.

3. M. Cardei, X. Cheng, X. Cheng, and D.-Z. Du. Connected domination in ad hoc wireless networks. In International Conference on Computer Science and Informatics (CSI), North Carolina, USA, March 2002. 
4. B. N. Clark, C. J. Colburn, and D. S. Johnson. Unit disks graphs. Discrete Mathematics, 86:165-177, December 1990.

5. T. Clausen and P. Jacquet. Optimized link state routing protocol (OLSR). RFC 3626, IETF, October 2003.

6. M. Jiang, J. Li, and Y. C. Tay. Cluster based routing protocol (CBRP). Internet draft version 01, IETF, July 1999.

7. C. R. Lin and M. Gerla. Adaptive clustering for mobile wireless networks. IEEE Journal of Selected Areas in Communications, 15(7):1265-1275, 1997.

8. C. E. Perkins, E. M. Belding Royer, and S. R. Das. Ad hoc on-demand distance vector (AODV) routing. RFC 3561, IETF, July 2003.

9. F. Theoleyre and F. Valois. A virtual structure for mobility management in hybrid networks. In Wireless Communications and Networking Conference (WCNC), Atlanta, USA, March 2004. IEEE.

10. F. Theoleyre and F. Valois. About the self-stabilization of a virtual topology for self-organization in ad hoc networks. In LNCS, editor, Self-Stabilization Symposium (SSS), volume 3764, Barcelona, Spain, October 2005. IEEE.

11. F. Theoleyre and F. Valois. Virtual structure routing in ad hoc networks. In International Conference in Communications (ICC), Seoul, Korea, May 2005. IEEE.

12. J. $\mathrm{Wu}$ and $\mathrm{H}$. Li. Dominating-set-based routing in ad hoc wireless networks. In International Workshop on Discrete Algorithms and Methods for Mobile Computing and Communications (DIAL'M), Seattle, USA, August 1999. ACM. 